\title{
Artificial Intelligence, System Analysis and Simulation Modeling in Precise Prediction of 5-Year Survival of Esophageal Cancer Patients after Complete Esophagogastrectomies
}

\author{
Oleg Kshivets \\ From World Society of Cardiothoracic Surgeons 25th Anniversary Congress, Edinburgh \\ Edinburgh, UK. 19-22 September 2015
}

\section{Background/Introduction}

Modern TNM-classification is based only on cancer characteristics and does not take into account at all the features of extremely complex alive supersystem - the patient's organism.

\section{Aims/Objectives}

We examined factors in terms of precise prediction of 5 -year survival (5YS) of esophageal cancer (EC) patients (ECP) (T1-4N0-2M0) after complete (R0) esophagogastrectomies (EG).

\section{Method}

We analyzed data of 491 consecutive ECP (age $=56.2 \pm$ 8.8 years; tumor size $=6.3 \pm 3.4 \mathrm{~cm}$ ) radically operated and monitored in 1975-2015 $(\mathrm{m}=359, \mathrm{f}=132$; EG Garlock $=280$, EG Lewis $=211$, combined EG with resection of pancreas, liver, diaphragm, aorta, VCS, colon transversum, lung, trachea, pericardium, splenectomy $=147$; adenocarcinoma $=279$, squamous $=202$, $\operatorname{mix}=10 ; \mathrm{T} 1=90, \mathrm{~T} 2=112, \mathrm{~T} 3=166, \mathrm{~T} 4=123 ; \mathrm{N} 0=$ $227, \mathrm{~N} 1=69, \mathrm{~N} 2=195 ; \mathrm{G} 1=136, \mathrm{G} 2=123, \mathrm{G} 3=232$; early $\mathrm{EC}=71$, invasive $=420$; only surgery $=377$, adjuvant chemoimmunoradiotherapy-AT $=114: 5$-FU +thymalin/taktivin+radiotherapy 45-50 Gy). Multivariate Cox modeling, clustering, SEPATH, Monte Carlo, bootstrap and neural networks computing were used to determine any significant dependence.

\section{Results}

Overall life span was $1776.1 \pm 2223.2$ days and cumulative 5 -year survival (5YS) reached $47.1 \%, 10$ years - 40.3\%, 20 years - 30\%. 147 ECP lived more than 5 years, 79 10 years. 223 ECP died because of EC. Cox modeling displayed $(\mathrm{Chi} 2=293.38, \mathrm{df}=18, \mathrm{p}=0.000)$ that $5 \mathrm{YS}$ of ECP significantly depended on: phase transition (PT) N0-N12 in terms of synergetics, cell ratio factors (CRF) (ratio between cancer cells and blood cells subpopulations), T, G, age, AT, localization, blood cells, prothrombin index, coagulation time, residual nitrogen $(\mathrm{p}=0.000$ 0.014 ). Neural networks, genetic algorithm selection and bootstrap simulation revealed relationships between $5 Y S$ and PT N0-N12 (rank = 1), T, AT, G, prothrombin index, glucose, blood cells, localization, PT early-invasive EC, CRF. Correct prediction of 5 YS was $100 \%$ by neural networks computing.

\section{Discussion/Conclusion}

$5 Y S$ of ECP after radical procedures significantly depended on: 1) PT "early-invasive cancer"; 2) PT N0-N12; 3) CRF; 4) blood cell circuit; 5) biochemical factors; 6) hemostasis system; 7) adjuvant chemotherapy; 8) tumor localization.

Published: 16 December 2015

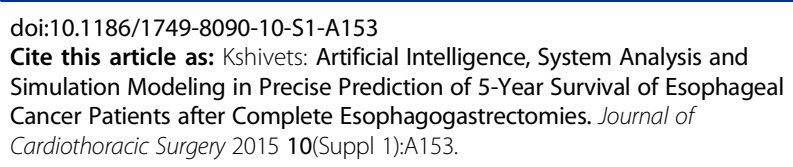

